\title{
Plumbemia en Niños de la Ciudad de La Plata, Argentina y su Relación con la Deficiencia de Hierro y los Factores de Exposición al Plomo
}

\author{
Bioq. LILIANA DISALVO ${ }^{1}$, Lic. CLAUDIA AAB ${ }^{1}$, Bioq. SILVIA PEREYRAS ${ }^{1}$, Bioq. JORGELINA PATTÍN ${ }^{1}$, Lic. \\ MARÍA APEZTEGUÍA ${ }^{1}$, Dr. JUAN CARLOS IANNICELLI ${ }^{1}$, Dra. ANA GIRARDELLI ${ }^{1}$, Bioq. ANA VAREA ${ }^{1}$ \\ 1. Instituto de Desarrollo e Investigaciones Pediátricas (IDIP) "Prof. Dr. Fernando E. Viteri", del Hospital de Niños de La Plata, \\ (MS/CIC-PBA).
}

\section{SUMMARY \\ Blood lead levels in children from the city of La Plata, Argentina. Relationship with iron deficiency and lead exposure risk factors}

Introduction: Environmental exposure to lead and the subsequent poisoning are a main public health concern worldwide. Children have a higher vulnerability to lead toxic effects, and many reports have shown the association between iron deficiency and lead poisoning. In Argentina, reports about lead levels in children are scarce. Our aims were to assess blood lead levels in children and determining their relationship with iron deficiency and known lead exposure risk factors. Material and Methods. We performed a crosssectional study in a sample of 93 children (age range: 6 months to 5 years) receiving care at La Plata Children's Hospital. A social and environmental survey was done, and blood lead, hemoglobin and ferritin levels were assessed. Results: Geometric mean blood lead level was $4.26 \mu \mathrm{g} / \mathrm{dl}$ (95\% CI, 3.60-5.03); prevalence of blood lead levels $\geq 10 \mu \mathrm{g} /$ dl was $10.8 \%$. Higher blood lead levels were found in children living in households with lead-handling contaminating activities $(6.74 v s 3.78 \mu \mathrm{g} / \mathrm{dl} ; \mathrm{p}=0.005)$ and in very low-income households $(5.68 v \mathrm{~s} 3.71 \mu \mathrm{g} / \mathrm{dl} ; \mathrm{p}=$ 0.020 ). The presence of blood lead levels $\geq 10 \mu \mathrm{g} / \mathrm{dl}$ was strongly associated with iron deficiency (OR 5.7; 95\% CI: 1.34-23.41) and with lead-handling activities at home (OR 4.8; 95\% CI: 1.12-20.16). Conclusion: The prevalence of blood lead levels $\geq 10 \mu \mathrm{g} / \mathrm{dl}$ is a matter of concern in the population studied. Iron deficiency and development of lead-handling activities at home were the risk factors associated with high blood lead levels. (Key words: lead poisoning, iron deficiency, environmental exposure, children).

Arch Argent Pediatr 2009; 107(4): 300-306

Correspondencia a:

Bioq. Liliana Disalvo.

E-mail: lilianadisalvo@yahoo.com.ar 


\section{RESUMEN}

Introducción: La exposición ambiental al plomo constituye un problema de salud pública en todo el mundo y los niños son más vulnerables a sus efectos tóxicos. Numerosas publicaciones demuestran que la deficiencia de hierro y la intoxicación por plomo pueden asociarse, pero en la Argentina los estudios publicados sobre población pediátrica son escasos. Nuestro objetivo fue establecer la plumbemia en niños y determinar su relación con la deficiencia de hierro y con factores de exposición. Población, Material y Métodos: Se realizó un estudio transversal en 93 niños (6 meses - 5 años) que concurrieron al Hospital de Niños de La Plata para controles de salud. Se aplicó una encuesta socioambiental y se determinaron las concentraciones de plomo, hemoglobina y ferritina en sangre. Resultados: La media geométrica de plomo en sangre fue 4,26 $\mu \mathrm{g} / \mathrm{dl}$ (IC 95\%: 3,60-5,03), con una prevalencia de plumbemias $\geq 10 \mu \mathrm{g} / \mathrm{dl}$ de $10,8 \%$. Se encontraron concentraciones de plomo más elevadas en los niños en cuyos hogares se desarrollaban actividades contaminantes $(6,74$ contra $3,78 \mu \mathrm{g} / \mathrm{dl} ; \mathrm{p}=0,005)$ y en quienes habitaban en viviendas precarias $(5,68$ contra $3,71 \mu \mathrm{g} / \mathrm{dl} ; \mathrm{p}=0,020)$. Las plumbemias $\geq 10 \mu \mathrm{g} / \mathrm{dl}$ se asociaron significativamente con la deficiencia de hierro (OR: 5,7; IC 95\%: 1,3423,41) y con la actividad domiciliaria contaminante (OR: 4,8 IC 95\%: 1,12-20,16). Conclusión: La prevalencia de plumbemias $\geq 10 \mu \mathrm{g} / \mathrm{dl}$ es preocupante en la población estudiada. Los factores de riesgo asociados a dichas concentraciones fueron la deficiencia de hierro y el desarrollo en el hogar de actividades relacionadas con la manipulación de plomo.

(Palabras clave: Plomo, deficiencia de hierro, exposición ambiental, niños).

ESTE TRABAJO LO PUEDE ENCONTRAR EN EXTENSO EN WWW.SciELO.ORG 